\title{
Les libations et le rôle de la présentatrice d'eau enfarinée dans le sacrifice mwaba-gurma (Nord- Togo)
}

Libations and the role of the woman who prepares floured water in Moba-Gurma sacrifices (Northern Togo)

\section{Albert de Surgy}

\section{OpenEdition}

Journals

Édition électronique

URL : http://journals.openedition.org/span/508

DOI : $10.4000 /$ span.508

ISSN : 2268-1558

Éditeur

École pratique des hautes études. Sciences humaines

Édition imprimée

Date de publication : 30 mai 1981

Pagination : 127-154

ISSN : 0294-7080

Référence électronique

Albert de Surgy, "Les libations et le rôle de la présentatrice d'eau enfarinée dans le sacrifice mwabagurma (Nord-Togo) », Systèmes de pensée en Afrique noire [En ligne], 5| 1981, mis en ligne le 02 juillet 2013, consulté le 30 avril 2019. URL : http://journals.openedition.org/span/508 ; DOI : 10.4000/ span.508 
I. E S L I B A T I O N S E T L E ROLE D E I A PRESENTATRIEEA'EAU ENFARINEE DAN S LE SACRIFICE MWABA-GURMA

$(N O R D-T O G O)$

par

Albert de Surgy

Trois termes sont utilisés par les Mwaba pour đésigner le sacrifice :

-- Le moins fréquent est doun qui fait référence au genou et à 1 'agenouillement, car le sacrifice provoque 1 a venue au monde d'un évènement qui ne se serait pas spontanêment produit, à la manière dont un bébé vient y faire son apparition hors du sein d'une parturiente agenouiliée.

-- Le terme le plus rigoureux est patr qui désigne en même temps 1 'action de gratter, déchirer, fouiller la substance invariante du sujet pour en faire sortir 1 'une des potentialitês, et la portion de terre pétrie, tenant lieu d'autel, qui représente cette substance ou celle de I'entité invisible (le destinataire du sacrifice) chargée d'aller prélever dans cette substance, à laquelle aucun vivant n'a directement accès, la richesse divine demandée.

-- Mais le terme de loin le plus utilisé est celui de "versement d'eau" qui désigne tout acte de relation avec le monde divin, qu'il $\mathrm{s}^{\prime}$ agisse de la prière -- qui est simple versement d'eau -- ou du sacrifice proprement dit -- qui est un versement d'eau accompagné d'un animal.

La substance du sujet (ou celle de l'entité invisible intermédiaire qui sert à l'atteindre), vers laquelle est dirigée l'action sacrificielle, pouvant être définie comme l'endroit où se rend l'eau du sujet, la coexistence est si étroite entre l'eau et le patr que celui-ci se laisse parfois désigner par le mot "eau" (1).

Le sacrifice mwaba se présente donc comme un moyen d'entretenir une relation avec une réalitê fondamentale, lointaine et cachée, pour 
en obtenir artificiellement le bénéfice de la production au monde de certains bienfaits qu'il faudra certes payer par des animaux, mais qui sont obtenus avant tout grâce à un versement d'eau.

Sans "versement d'eau", pas de sacrifice.

Sans immolation d'animaux, par contre, on accomplit déjà un acte religieux de 1 'odre du sacrifice :

-- soit en représentant des animaux par quelques parties de leurs corps (pattes de quadrupèdes et/ou plumes de poulet), pourvu que 1 'offrande symbolique qui en est ainsi faite soit accompagnée de libations de bière de mil. On procède gênéralement ainsi, avant de sacrifier réellement au profit de la personne qui en a besoin, pour s'acquitter de sacrifices initiatiques éventuellement négligés par des aĩeux, et 1 'on accomplit là un patr-kpel ou "ancien sacrifice".

-- soit en faisant seulement voeu d'un sacrifice en représentant aussi les animaux par des plumes, mais en ne versant cette fois que de l'eau simple. On accomplit alors un tompuk-patr, ou sacrifice (patr) pour demander pardon, en s'excusant de ne pouvoir exécuter pour le moment ce qu'on promet d'exécuter plus tard une fois satisfaction pleinement accordëe.

Il apparâtt ainsi que le versement de libations est plus essentiel au sacrifice que 1'immolation des animaux. Une théorie du sacrifice qui ne mettrait pas au premier rang le rôle de l'eau ne saurait donc nous en donner une interprétation satisfaisante.

Constatant une fascination irrationnelle des auteurs par le meurtre, la boucherie, la "bouffe", le sang, etc., quand de toute évidence (et pas seulement chez les Mwaba) 1'eau simple et la boisson importent davantage, je voudrais m'efforcer ci-dessous de combler quelque peu la grave lacune qui en rêsulte.

Mais pour mieux faire comprendre dans quel contexte l'eau intervient, il est utile de rappeler les principales conclusions qui-se dégagent à ce propos de l'êtude de la divination par les huit cordelettes à laquelle les Mwaba recourent systématiquement pour décider

(1) "Où est 1 'eau de l'enfant ?" s'interrogera le consultant ou le devin en cherchant à identifier 1 'ancêtre de même principe spirituel qui parraine 1 a venue de 1 'enfant au monde et servira de médiateur pour en atteindre la substance. 
de 1 'opportunité ou des modalités d'un sacrifice (2).

1. La parole d̀ l'origine de toute chose et, bien entendu, du sacrifice.

Selon la conception mwaba, tout ce que nous connaissons, tout évènement que nous ressentons, préexiste sous forme de signes ou de "paroles" intérieures radicalement inconscientes, en un lieu visualisé en conséquence dans les entrailles inaccessibles de la Terre.

Ces paroles, proférées par la Mère du monde, constituent l'univers référentiel de tous les possibles, celui des essences ou des formes pouvant être réalisées chacune en une multitude d'exemplaires distincts ; il n'est rien de concret dans le monde qui ne trouve parmi elles son élément d'identification et comme son nom.

En particulier, toute expérience vécue de l'home préexiste en sa substance sous forme de virtualité inclue par lui dans son domaine du temps oì il vivait sous le contrôle de Dieu dans la demeure de la Mère du monde ; et toutes les virtualités inscrites ainsi en sa substance tendent à se râaliser fatalement dès que 1'occasion s'en présente.

Une bonne part des maux dont souffrent les hommes sont dus au choix malencontreux qu'ils ont fait de mauvaises virtualités ; et seul le Dieu créateur, artisan de l'origine, a pouvoir de les en sauver. Alors que les sacrifices ordinaires se proposent en quelque sorte de tirer le meilleur parti du matériel génétique de l'individu, les cérémonies de purification de telles virtualités ont 1'aspect d'une manipulation de ce matériel génétique par mise à la masse du potentiel de certains de ses éléments.

Par ailleurs, tout sacrifice est accroché à la parole de l'origine que le travail du devin consiste à découvrir en fouillant symboliquement la substance comme un cultivateur travaille la terre. Il n'est

(2) Comme il est loin d'être rare en Afrique noire occidentale que des consultations régulières de devins, parfaitement approuvées par tous, conduisent à des sacrifices, et comme de telles consultations mettent spontanément en relief les raisons qui décident ou non de les prescrire, il me semble bien téméraire de vouloir traiter des sacrifices dans cette région du monde en faisant l'économie de l'étude de la divination qui en révèle toute la gamme, et le pourquoi et le comment. Toute interprétation élaborée en se fermant ainsi lés yeux sur les conceptions indigènes qui justifient et engendrent les pratiques considérées me parầt suspecte. 
pas de sacrifice qui ne tienne ainsi à une parole et ne débute par une affirmation du genre : "les jabab (devins) m'ont dit que ...".

Une phase importante de la consultation divinatoire consiste en un travail rituel de la parole abstraite initialement recueillie pour la transformer en une parole particulière que s'appropriera le consultant et à laquelle il accrochera un sacrifice pour que le germe qu'elle représente alors porte fruit.

Le premier terme du sacrifice est de ce fait posé dès la dernière phase de la consultation divinatoire, et l'on ne saurait manquer de le reconnâ̂tre déjà là sans être conduit à des erreurs d'interprétation. Même le rôle de l'eau sur lequel je vais insister lui reste subordonné, car 1 'eau ne sert à rien si ce n'est à provoquer la germination de quelque chose qui doit être dêcouvert et livré au consultant par le devin.

Ajoutons que toute parole oraculaire, préfiguration de toute réalité élémentaire, est constituée par un partage en trois de 1 'ensemble des huit cordelettes, qui fait apparâ̂tre deux groupes latêraux de trois cordelettes assimilés à deux "querelleurs" et un groupe central de deux cordelettes assimilé à l'arbitre et au juge, qui définit la position d'équilibre des deux premiers. En effet, le germe de toute chose est constitué d'un terme d'identification fixé par Dieu (yêdu, le tagdấ ou "mâttre de la création" qui a composé la substance première du monde) associé à deux puissances contraires agissant l'une dans le sens femelle de la procréation et de la matière (tag-pwo ou la femelle de la création) et l'autre dans le sens mâle du maintien, ou du retour, à l'origine et de la forme soumettant la matière (tag-jwa ou le mâle de la création).

La venue au monde, ou procession des réalités, résulte d'une prêdominance de Ia première; et le retrait du monde, ou conversion des réalités à leur principe, comme tout mouvement pour atteindre le principe, est commandé par le second. 


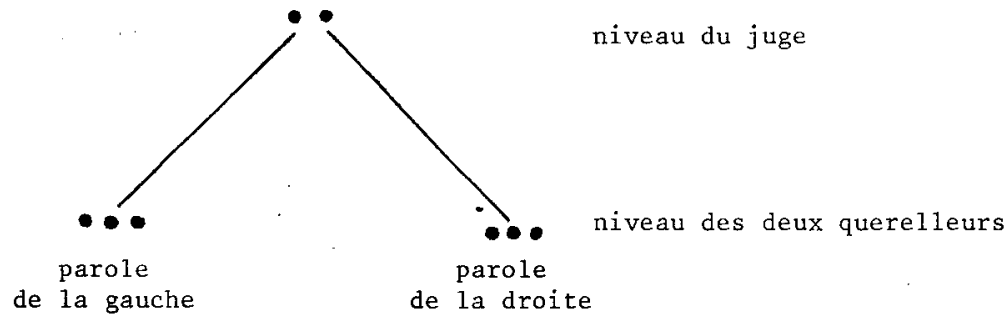

Structure de toute parole oraculaire, ou parole intêrieure rêférentielle, inscrite dans la substance première (tagm)

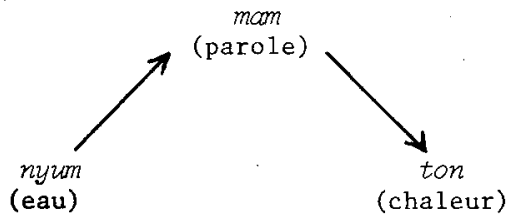

Les trois noms de la cordelette divinatoire suprême pouvant représenter 1 'ensemble des cordelettes

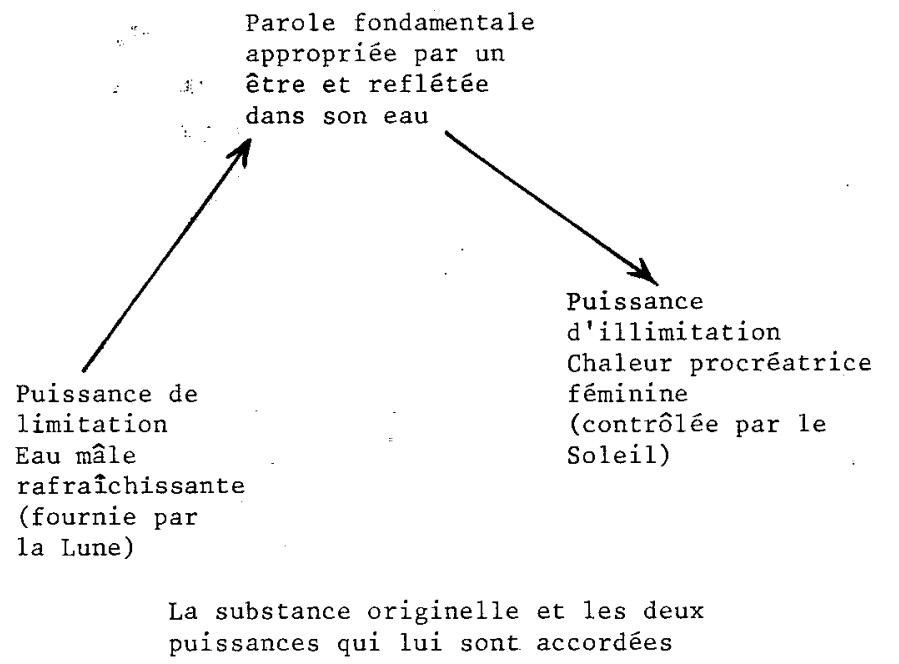


Seuil du monde divin

Niveau de la transcription des idêes

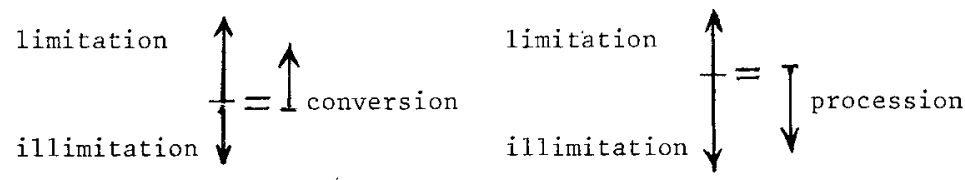

Seuil du monde visible

Niveau de lecture des messages en vue de leur traduction en réalités visibles ou sensibles

Excès de frâ̂cheur

L'idée fait retour

à son entrepôt
Excès de chaleur

L'idée vient se concrétiser dans

le monde

2. Le monnayage par des animaux sacrificiels de ce qui empêche un bienfait espéré de venir au monde

Dieu veille au bon ordre du monde en empêchant tous les germes des réalitês visibles qu'il a créés d'entrer en activité tous à la fois. Cette mise en activité des germes est soumise au contrôle des rythmes astronomiques d'une façon que le paysan comprend fort bien puisqu'il est un temps propice à chaque semis. Ce contrôle permet à la création, toute entière conçue à 1'avance, d'être déroulée avec ordre, petit à petit.

Un tel gouvernement par les rythmes astronomiques du débouché du sein de la Terre pour qu'y reste tenu caché ce qui ne doit en sortir qu'en son temps, est assimilé à la mise en poste, autour de la demeure de la Mère du monde, de puissances gardiennes d'origine cêleste que le Dieu-Soleil, roi du monde terrestre, et fils unique de cette Mère, se permet impunément d'aller dérober au ciel, hors de chez lui, et de réduire en esclavạge (il est alors qualifié de yom'dââ,"mâ̂tre d'esclaves") (3). 
La demeure divine souterraine, d'où toute richesse provient au monde en suivant le même trajet est-ouest que le solei1, apparaît ainsi défendue par un certain nombre de "garçons", et pour y prélever le germe d'une réalité désirée mais qui ne vient pas spontanément au monde, il faut commencer par vaincre un (ou plusieurs) de ces "garçons".

\section{1. La première immolation : celle de za puissance gardienne.}

L'opération sacrificielle débute donc par le meurtre ou le démantèlement de la puissance mâle qui maintient en profond sommeil dans la demeure des âmes la plupart des richesses de la création.

Ce meurtre, l'homme lui-même est incapable de l'accomplir. Ce sont des êtres intermédiaires entre le monde des vivants et le monde de l'origine qui l'accomplissent en son nom, soit à la demande qui leur

(3) Cette domination par le Soleil des puissances intellectuelles extërieures au monde dont il est roi, mais. nécessaires à la mise en ordre de celui-ci, reçoit, semble-t-il, une explication mythique sur laquelle nous manquons d'éléments décisifs et proposons done à titre d'hypothèse :

I1 y eut à 1 'origine la vaste cour circulaire du ciel, peuplée d'une grande famille, et la cour carrée du dieu Terre qui vivait seul privé de descendance.

L'une des filles du ciel fut accordée en mariage au dieu Terre. Mais comme la lumière dévore les ténèbres, et comme la mante religieuse qui est son symbole dévore le mâle après 1 'accouplement, sitôt fêcondée par lui, elle le dévora et devint ainsi l'unique souveraine du monde.

Des rapports qu'elle eut avec le dieu Terre elle engendra par 1 'extrémité inférieure de son corps un seul couple de jumeaux : le Soleil et la Lune. Cependant, par l'extrémité supërieure de son corps, fruits de son imagination mais non de son sexe, furent proférées, sous forme de "paroles", toues les idées susceptibles de prendre corps dans le monde visible.

Ayant cédé sa soeur en mariage au lignage de son oncle maternel, c'est à dire au ciel, le Soleil demeura lui-même cêlibataire et se plaça exclusivement au service des oeuvres conceptuelles de sa Mère pour lui assurer tout de même, de façon originale, une continuation. (Bien que les missionnaires le confondent volontiers avec le Dieu unique des chrétiens, le célibat de ce Dieu-Soleil, yêdu, est son seul caractère d'unicité, confronté qu'il est à sa mère, à sa soeur et aux membres du lignage du. Ciel).

C'est en compensation de la multitude des enfants que sa soeur la Lune produisit 1 à-haut sous forme de corps cêlestes qu'il acquit le droit de monter y dérober tout ce qui lui plaisait, y compris la plupart des"maris" de sa soeur, pourvu qu'il en reste au moins un, tour à tour, auprès d'elle pour la féconder. 
est faite (par un tompuk-patr, évoqué plus haut), soit spontanëment pour venir au secours des vivants qu'ils protègent quand ces derniers n'ont pas eux-mêmes conscience d'être menacés.

Cette première immolation a lieu dans le secret de I'invisible. Ce n'est que par un devin que $1^{\prime}$ homme apprend qu'elle a été accomplie et qu'il en résulte pour lui une dette sacrificielle à l'égard de 1 'entité qui est intervenue à son profit.

Le sacrifice mwaba-gurma $n$ 'est jamais accompli pour contraindre une entité spirituelle à agir en faveur des hommes et déclencher automatiquement un afflux de grâces divines, mais toujours pour rembourser symboliquement une entité spirituelle des risques qu'elle a assumés en êtant dẻjà effectivement intervenue.

On ne $s$ 'attaque pas en effet impunëment à une puissance gardienne placée par Dieu à 1'orifice des entrailles de la Terre. A la suppression de 1 'équilibre assurê par son contrôle mâle fait suite un afflux de chaleur procréatrice qui dérange en premier lieu l'entité spirituelle qui est intervenue et que celle-ci répercute directement ou de proche en proche sur son protégé.

"Son père (dëfunt), c'est que ça l'a chauffé dans le môdâa (ancêtre tutélaire) de sa fille (après que le mâdâa soit intervenu au profit de celle-ci). Comme ça $1^{\prime}$ a chauffé il a promis (au nom de la fille, à son $m \hat{a} d a \hat{a})$ un chien et une chèvre ; et le mâda ât àène le bruit (pour appeler $1^{\prime}$ attention sur le sacrifice à acquitter). C'est son $m \hat{a} d a \hat{a}-b u l \hat{k}$ qui lui amène toujours la chaleur (enregistré chez Lasembet Kolani, à Doré, le 26/11/77).

Si 1'individu néglige de payer par un sacrifice 1 'intervention qui a eu lieu en sa faveur, il est bientôt menacé de mort, car le meurtre d'une puissance gardienne doit être compensé par le sacrifice d'une vie pour que le monde retrouve après coup I'équilibre et la douceur ; car 1 'obligé doit reconnâtre par un sacrifice les liens de soumission hiérarchique qui 1 'unissent aux entités qui le mettent en communication avec sa source de vie et sans la médiation bienveillante desquelles il n'existe plus. En effet, les consultations divinatoires faisant suite à un dëcès ont pour but principal d'identifier l'entité invisible qui a éventuellement causé la mort du sujet pour n'avoir pas été honorée, selon son droit, d'un sacrifice.

En d'autres termes, la brèche ouverte dans le rempart de la demeure divine, où dorment toutes les richesses dont peut bénéficier le monde, doit être bouchée, si l'on veut éviter la souffrance, par 
1a mise en place d'une puissance gardienne équivalente à celle qui en a été écartée.

2. 2. La seconde inmolation: celle d'un poulet et d'un quadrupède.

Le "garçon" céleste établi par le Dieu-Soleil comme gardien était caractérisé par le nombre six : celui mis en évidence par ses quatre membres, sa tête et son sexe; et celui de la subdivision en six des trente-six unitês de la circonférence céleste.

En conséquence doivent être envoyés à sa place un poulet et un quadrupède (ou l'équivalent d'un quadrupède) totalisant ensemble six pattes.

Une fois morts, le poulet et le quadrupède, sortis du vestibule ou de la périphérie de la demeure des hommes, s'identifient symboliquement à la puissance gardienne qu'ils équivalent comme une sorte de monnaie. Et tandis que leur forme, ou leur être dématérialisé réduit à ce qu'il signifie, prend position autour de la demeure divine en la ceinturant comme d'une sorte de zodiaque (zodion signifiant en grec petit animal), le dépeçage, la cuisson et la consomation de leurs corps célèbrent le démantèlement de la puissance gardienne originelle, 1a procession en direction du monde des hommes, sous 1 'empire de la chaleur procréatrice, des parties de son corps démantelé, et enfin sa reconstitution autour du monde des hommes, à la suite de son incorporation par les divers représentants de l'unité du corps social, sous forme d'un corps de significations et de valeurs assurant la participation de tous à une même culture.

Il y a de la sorte une permutation entre des vies animales qui entouraient la demeure des hommes et des puissances gardiennes qui entouraient la demeure divine; cependant que le traitement du corps des victimes, par suite d'une transsubstantiation qui les assimile au corps de la puissance gardienne elle-même, a représenté 1'abaissement de celle-ci jusqu'au monde des hommes pour faire de ceux-ci des créatures supérieures aux animaux, ayant immédiatement accès à 1 'ordre intellectue1.

On voit là célébré par le sacrifice infiniment plus que le surcrô̂t d'enfants, de nourriture, de santé, etc., innocemment demandé aux ancêtres ou aux gểnies. 
3. L'humidification indispensable de la substance.

Une fois que l'accès aux richesses divines a été dégagé par suppression d'une puissance mâle, encore faut-il que ces richesses parviennent au monde.

or, on ne rend pas visible $1^{\prime}$ invisible et rien de ce que contient la demeure divine ne peut en sortir : il s'agit là d'un univers référentiel invariant qui contient tous les possibles et qu'on ne saurait priver de rien, à jamais retranché de toute participation au devenir matêrie1.

Seules des copies d'un tel univers peuvent venir au'monde. I1 convient donc d'assurer la transcription des messages qui y sont contenus, puis la projection de ces messages, sous forme de paroles créatrices, dirigées vers le monde pour qu'elles y soient entendues et exécutées.

Qu'il s'agisse de la production spontanêe ou sacrificielle des évènements, l'indispensable fonction de transcription est assurée par 1 'eau. Cette eau imbibe la substance de 1 'homme ou du monde, se laisse imprégner des paroles intérieures ou signes inscrits en cette substance et en garde le reflet ; puis chauffée par le soleil créateur qui donne vie au monde, elle se transforme en vapeur et "souffle" $1 \mathrm{a}$ parole qu'il faut exprimer à travers la bouche de la Terre jusqu'au monde visible. Au seuil du monde visible, elle se condense à nouveau en déclenchant alors la production des phénomènes matériels.

Ainsi naquit le monde, mélange boueux de terre et d'eau à 1 'origine, avant que l'évaporation causêe par le Soleil ne solidifiât une portion de croûte terrestre purgée de paroles, appelée à servir de socle et de docile fournisseuse d'éléments matériels aux formes vivantes.

Ainsi naît d'instant en instant la vie qui nous anime pour autant que nous sommes autre chose qu'une mécanique livrée à 1 'enchânement des causes et des effets et sommes l'expression d'une âme d'où nous est accordée une eau de jouvence.

Ne pas acquitter la dette d'eau qui en résulte, ne pas tenir sa substance constamment humidifiée en y faisant parvenir de l'eau, par l'intermédiaire des entités spirituelles protectrices qui savent où l'atteindre, est non seulement se priver du bénéfice de la transcription 
et du don de tous les vrais trésors de la vie, répliques de ceux du monde divin, mais encore s'exposer, au lieu de recevoir de l'origine un vent de frâ̂cheur, à être désséché et enfièvré par un vent de plus en plus chaud, puis pour finir par une absence de vent, une rupture du souffle qui cause immédiatement la mort.

Bref, on pourrait offrir des animaux, les égorger, se les partager, les consommer, et en offrir une part sur les autels, ce serait parfaitement en vain s'il n'était aussi versé de l'eau. Contrairement à toute attente, il n'en résulterait éventuellement qu'un afflux de chaleur !

\section{Les trois principales variétés de libations. (4).}

L'eau se présente sous trois grands aspects, ou trois phases, selon qu'elle reflue vers l'origine, selon que, appliquêe à l'origine, elle s'imprégne des signes ou idées contenus dans la demeure divine, ou selon que, rétrogradant sous l'effet de la chaleur procréatrice, elle accompagne les paroles qui expriment au dehors ces idées.

a) Sous 1'aspect d'eau simple (nyu-kaal), eau mâle, elle est faite pour annuler la chaleur et en préserver l'individu en la reconduisant à sa source. Puissance de limitation par excellence, elle progresse jusqu'au seuil de la demeure divine pour tenir la substance de 1 'homme humidifiêe, lave et rafrâ̂chit au passage toute la voie qui y conduit, et emporte alors avec elle, à travers les mêmes échelons que ceux gravis lentement par les ancêtres, les paroles de supplication, qui sont des paroles "mortes", de l'orant.

Elle est versée pour accompagner toute prière, que celle-ci précède immédiatement ou non 1 'exécution d'un sacrifice.

b) Sous l'aspect d'eau tenant en suspension des particules de farine de mil (yô-nyum), elle représente l'eau enrichie d'une multitude d'essences, chacune d'elles rêduite au point sans substance qui l'identifie; l'eau primordiale de la crêation divine, chargẻe d'âmes

(4) Il en existe une quatrième : celle de lait mélangée à du miel, offerte en cadeau aux petits jumeaux invisibles qui apprécient les mêmes douceurs que les enfants, soit pour leur demander de laisser les vivants tranquilles, soit pour qu'ils aient satisfaction dans 1 'effort qu'on les incite à fournir. 
et de formes célestes en attente d'être envoyées prendre corps dans le monde visible; le liquide séminal versê au seuil de la demeure divine pour que puissent être manifestêes au dehors les conceptions qui y restent enfouies et que la Terre soit ainsi rendue féconde à la façon dont le sein de l'épouse est fécondê par le sperme de son mari (5). Une libation de cette eau-là n'est versée qu'à l'occasion de sacrifices sanglants dont on attend non seulement une prêservation de la chaleur, mais aussi la venue au monde de quelque bienfait. E1le atteste non plus un simple désir de protection mais un dêsir de communication et d'échange dont elle constitue le premier terme d'invite (6).

c) Sous I'aspect d'eau activée par le feu, chargée de tous les éléments qui 1 'ont transformée en bière de mil, daom, elle représente la puissance de limitation en train de céder à l'ivresse qui l'emporte jusqu'au monde et communiquant elle-même cette ivresse aux hommes, attachée aux paroles vivifiantes dont la réalisation vient d'être dêclenchée.

Si de la bière de mil n'est pas immédiatement versée en troisième lieu sur l'autel ou devant 1'autel (7), on ne manque généralement pas d'en donner à consommer aux bénéficiaires du sacrifice et d'en distribuer, si possible, aussi à tous les participants (bénéficiaires eux aussi d'une autre manière) au moment de leur distribuer de la nourriture sacrificielle ou de répartir entre eux les chairs des victimes.

Le cas de $1^{\prime}$ eau simple et de la bière de mil est sans mystère, mais celui de l'eau enfarinee ne 1 'est point, car c'est elle qui représente l'eau primordiale imprégnée des principes célestes sur lesquels prend appui toute projection de paroles vers le monde.

(5) Le trajet suivi par les paroles originelles, depuis la bouche de la demeure de la Mère du monde jusqu'au plan de leur réalisation, est analogue à l'évolution du futur être humain dans le sein de sa mère, de la fécondation de l'ovule à l'accouchement.

(6) Alors que 1 'eau simple est offerte au visiteur avec qui 1 'on ne souhaite pas entrer en amitiê, l'eau enfarinée est, au contraire, offerte en signe d'ouverture du coeur avant d'engager une discussion accompagnée si possible de consommation de bière de mil.

(7) Il arrive que de la farine soit directement délayée dans de la bière de mil versée juste avant l'immolation. 
Pour comprendre la nature de ces principes et la façon dont ils interviennent pour assurer l'acheminement jusqu'aux hommes des bienfaits attendus du sacrifice, il nous faut considerer les conditions dans lesquelles le monde put être procrêe (et continue d'être procrêê) compte tenu des conceptions déjà admises, car il le fut (et continue de l'être) hors d'une ouverture de la demeure divine de la même manière que le sont de tels bienfaits une fois écartée la puissance gardienne qui en interdit l'émission.

\section{La Lune, première dispensatrice de l'eau.}

Le paysan mwaba constate que 1 'eau propre à féconder son champ lui est apportée par le vent d'est à la suite d'une intense période de chaleur qui l'a évaporée de 1 'endroit où, originellement, elle avait été répandue.

Cet endroit d'où, à travers 1 'atmosphère, nous est accordée la vie n'est autre que le tagm ou substance première placée sous le contrôle du Dieu-Soleil. Or il s'agit là d'une substance terreuse brûlante qui resta privêe, à l'origine, du bénéfice de l'eau.

De même que 1 'eau qui donne vie au territoire du village lui est apportée de 1 'extérieur par le vent, 1 'eau primordiale qui féconda 1a substance originelle y fut, juge-t-on, apportée en provenance d'un lieu semblablement distant du monde $d$ 'en bas, depuis donc les confins du ciel... Avec l'eau qui, de l'avis gênéral, en est la demeure première, 1'âme descendit ainsi du ciel, caractêrisée par la portion de ciel d'où elle provenait.

Or non seulement 1 'eau, une fois transformée en vapeur pour vêhiculer jusqu'au monde les paroles créatrices, porte en elle un reflet d'idêes sélectionnëes dans 1 'univers de tous les possibles imaginés par la Mère du monde, mais aussi le reflet d'une moitié de la voûte céleste étendue au-dessus d'elle au moment de son évaporation.

A toute "parole" ëmise est ainsi attachée une caractéristique additionnelle en rapport avec le ciel, et plus précisêment avec une série de demeures des âmes, ou avec l'une quelconque de ces demeures placée en tête de celles qui suivent. Nous pouvons la considérer comme une qualification de la vapeur porteuse, indépendante des paroles 
vêhiculées, par laquelle est dêfini un ensemble ordonné de catégories de souffles ou d'esprits mis cycliquement à la disposition de tout ce qui doit être acheminé jusqu'au monde.

Tout le problème est de savoir combien de telles catégories, et parallèlement combien de demeures originelles des âmes, prendre en considération.

Le cadre de cette article ne nous permet pas d'en préciser la disposition exacte sur 1 a voûte du ciel, car elle est assez complexe pour être en accord avec le rythme du jour et de l'année (8) ; mais nous n'en dresserons pas moins l'inventaire.

Il est en effet une connection, qui n'échappe à personne, entre le rythme lunaire et la période menstruelle de 1a femme,comme avec la durêe de sa gestation estimée à 9 lunaisons. Dans 1'esprit du Mwaba, quelque chose se renouvelle à chaque lune au niveau du seuil de la demeure divine avec lequel est en correspondance le sein de la femme. Ce quelque chose n'est évidemment pas le reflet momentané du ciel ; il préside non pas à la manifestation dans le monde de telle ou telle parole choisie par l'âme, mais à la manifestation dans le monde de cette âme elle-même. Il permet donc de classer les âmes en catégories ou, en d'autres termes, de les regrouper par demeures identiques déployées de quelque façon en éventail sur la voûte du ciel.

or, si à chaque lune des âmes de même catêgorie sont envoyées prendre le départ du chemin qui les conduira à la naissance, cela implique que le principe lunaire, ou la déesse lune, assure à chaque lunaison le transfert de l'eau céleste où baignent les âmes jusqu'à 1 'une des ouvertures de la demeure divine au sein de laquelle elles s'attribuent, avant d'être soufflées au monde, les paroles composant leur programme prénatal.

De cette déesse nous n'apercevons jamais que le dessous de la calebasse avec laquelle elle assure ce transfert : il importe en effet que son contenu reste tout au long protégé du rayonnement solaire qui ne manquerait pas d'en altêrer la fraîcheur. Elle la remplit à la voûte du ciel, au moment de la pleine lune, dans la

(8) On trouvera plus ample information à ce sujet dans 1 'ouvrage que je prépare sur la divination mwaba-gurma. 
partie la plus frâ̂che du ciel en opposition diamétrale avec le soleil, puis descend peu à peu vers la demeure souterraine du dieu-Soleil et de sa mère, où elle arrive en période de nouvelle lune quand, ayant rejoint la nuit le Soleil, on ne la voit plus briller entre Terre et Ciel. Après en avoir versé le contenu, elle remonte aussitôt au ciel, et ainsi de suite...

De même que toute femme entretient la communication entre l'habitation à qui elle a été cédée en mariage et son habitation d'origine en allant périodiquement rendre visite à cette dernière (9), il revient à la Lune d'établir la communication entre le ciel et la terre (10). C'est parce que la Lune, modèle céleste de la femme, ne voyage jamais sans sa calebasse qu'il est recommandé à toute femme mwaba en voyage de porter en main une calebasse (12).

Quand il provoque 1'émission des paroles créatrices à travers la bouche de la Terre, le Soleil ne fait en somme que révéler les potentialités reflêtées dans 1 'eau sur laquelle il darde ses rayons; en sorte que la Lune, qui fournit l'eau, parâ̂t tout aussi indispensable que lui, sinon plus, au développement de la création ... et pareillement à la réussite du sacrifice.

De par la relation qu'elle entretient avec les entrailles de la

(9) Voir plus haut, note (3). Le ciel est sa demeure d'adoption et la terre sa demeure d'origine.

(10) Telle qu'il me paraît juste de la reconstituer, l'explication mythique de ces visites est la suivante :

Aussitôt dépossédée par ce voleur d'hommes célestes, ou propriétaire d'esclaves, qu'est le Soleil, du mari qui vient de la mettre. en grossesse, la Lune doit songer à descendre chez son frère récupérer celui de ses maris dont le tour est venu de remonter avec elle afin qu'elle soit pareillement."pleine" dès la lunaison suivante.

Aussitôt après avoir dégarni une ouverture de la demeure divine pour remporter avec elle le gardien qui y était placé, elle y déverse en compensation le contenu de la calebasse qu'elle a pris soin de remplir avant de descendre.

Cette eau fraîche empêche la chaleur infernale du monde d'en bas de monter déranger les êtres du Ciel ; et au lieu de la contraindre par la force, la réduit par la douceur en en tolérant néanmoins l'expansion sous la forme du grand jeu de la création.

(11) A 1'homme qui voyage il est recommandé de porter sa lance, symbole du "garçon" dont le Soleil est cette fois le modèle. 
femme, son rôle est célêbré à l'occasion de tout sacrifice sanglant par une femme ayant de préférence même principe spirituel (mięl), c'est à dire même souffle originel, que le bénéficiaire du sacrifice, cếdée en mariage à l'extérieur, mais rappelée par son frère à cette occasion. E1le est chargée de tenir en mains toute calebasse de libations, mais plus particulièrement de dêlayer la farine dans l'eau, symbole de l'eau céleste chargée d'âmes, qui doit être déversée sur les mottes de terre tenant lieu d'autels qui représentent à divers titres une part de la substance du monde.

Les particules de farine en suspension dans l'eau représentent parfaitement en effet, baignant dans 1'océan de la conscience universelle, des êtres éternels, purs de substance, d'une nature antérieure à celle des grains qui se constituent autour d'eux et par eux, et dont ils deviennent les points représentatifs. Appelés à germer et à devenir plantes, ces grains constituent déjà le premier terme du devenir, mais ces êtres antêrieurs êchappent eux-mêmes au devenir et les grains redevenus grains en fin de cycle les restituent une fois détruits.

Appelée sâââdâa, la "salvatrice" (12), ou pwotwa, cette femme qui prépare et présente l'eau enfarinée joue dans l'opération sacrificielle un rôle apparemment effacé, mais tout aussi important que l'officiant chargé d'envoyer la prière et d'égorger ou faire égorger les animaux.

Alors que le Soleil mâle agit dans le sens féminin de la procréation du monde, la Lune, femelle, agit dans le sens masculin qui est celui de l'étouffement par l'eau du feu procréateur, ou de la fourniture de liquide séminal, si bien que la sâdâdấ qui la représente est caractérisée par le nombre trois qui est mâle. En effet, dit-on, comme elle se tient à genoux en réunissant ses deux mains sous la calebasse qu'elle prêsente, "ses membres forment trois".

6. Les 33 puissances du "serpent de za création"

Puisque tout est réglé par l'activité de la Lune, il est autant

(12) Mot à mot "la propriétaire (dâa) de la supplication (sâdant)". On appelle ainsi toute personne sur laquelle monte se réfugier un enfant qui se sent menacé. 
de demeures célestes des âmes, autant de catégories différentes de souffles suscités par le Soleil qu'il y a de variêtés d'eau puisée par la Lune sur la voûte du ciel, autant donc que de localisations différentes de la pleine lune sur la voûte du ciel. Puisque le soleil met un an à parcourir le cercle du zodiaque, il y en a exactement autant que de lunaisons entières dans un nombre entier d'années.

Pour bien mettre en valeur les propriêtés de ce nombre, remarquons d'abord qu'il est êgal au nombre de séries de lunaisons, dont chacune approche au plus près de l'année solaire, que 1 'on trouve comprises dans un nombre entier d'années solaires.

Or la série de lunaisons qui avoisine au plus près l'année solaire forme une "année lunaire" de 12 lunaisons à l'issue de laquelle sont jugées ouvertes, à tour de rôle, en un mouvement alternatif de va et vient, six catégories d'ouvertures de la demeure divine, ordonnées le long d'un axe en fonction de la force qu'il est nécessaire d'y appliquer pour y dominer 1 'ënergie des paroles prêtes à en sortir.

Ce sont là six "bouches" possibles de la terre, devant être obturées chacune par un gardien de puissance plus ou moins élevêe que le Soleil va voler dans une partie correspondante de la face invisible du ciel. Il leur correspond six poulets sacrificiels (13) destinés à arracher spécifiquement, là où ils se trouvent, la venue au monde de bienfaits qui n'y parviennent pas spontanément.

Etant donné qu'à chaque lunaison correspond un versement d'eau caractêristique sur 1 'une des ces "bouches", et une activité caractéristique complémentaire du Soleil, que nous considérions ces bouches isolément ou que nous les considérions dans leur ensemble; il existe autant de demeures célestes des âmes et de catégories de souffles que de façons pour une sërie de douze lunaisons de se présenter par rapport au cycle de 1 'année, c'est à dire autant que d'annếes lunaires différentes à l'intérieur d'une période commune luni-solaire au bout de laquelle le début de l'année lunaire, après avoir rétrogradé de 10,875 jours par an sur 1 'année solaire, finit par coincider de nouveau avec le même point de l'année solaire.

(13) Poulets blanc, noir, moucheté, rouge, cendré et bigarré. 
Ce nombre est en première approximation de 33 . En effet, c'est après 33 années lunaires qu'avec un écart par excès de 6,214 jours seulement (moins d'un quart de lunaison) le début de l'année lunaire revient rattraper le début de 1 'année solaire. (A ces quelques jours près, il s'est évidemment écoulé dans le même temps 32 années solaires).

La collection de tels principes du souffle, appelés parfois gbâgei (pluriel de gbâjok) en jargon divinatoire, a pour symbole la sërie des 33 vertèbres d'un grand python mythique connu sous le nom de tag-waog ou "serpent de la création" que représente à sa manière la seconde cordelette divinatoire, appelée ghấjok, identifiée par des vertèbres de python.

La tête de ce serpent représente 1'ensemble des conceptions de 1 'âme composant le discours intérieur qu'elle se tient et susceptibles de se réaliser au dehors. Etant donné le rôle directeur et mâle de la tête, plus spécifiquement représentēe par gb̂ajok, on la juge caractérisée par le nombre 3 et on lui attribue la dimension de 3 vertèbres.

Les vertèbres elles-mêmes qui constituent la cascade de principes par lesquels les conceptions contenues en la tête parviennent au monde sont plus spécifiquement représentées par la troisième cordelette divinatoire, appelée buzk, caractéristique par ailleurs du monde invisible des défunts.

A la suite de ces vertèbres est une queue qui représente ce qui, par-delà 1'étendue spirituelle proprement dite -- celle parcourue par le souffle porteur -- appartient déjà au monde visible sans être encore effectivement visible, et se situe au niveau de la recondensation de la vapeur en eau pour que soient épousées par la matière les conceptions destinées à y être inscrites. Elle représente ce qui, pardelà la conception purement spirituelle de l'homme, le place déjà en gestation dans le sein de la femme. En raison de cette relation au monde charnel et à la femme, on la représente par la même cordelette, appelêe katr, que la femme et on la caractêrise par le nombre 4, lui attribuant en conséquence une longueur de 4 vertèbres.

Au total le "serpent du créateur" possède donc une étendue mesurée par le nombre $3+33+4=40$. Et si 1 'on y ajoute 1 a langue, sortant de sa tête, représentée par la même première cordelette que les paroles, caractérisant toutes les conceptions possibles 
engendrées par la Mère du monde qu'il choisit d'avoir en tête, cela lui donne une étendue mesurée par le nombre 41 (14).

Cependant, tenu au repos au seuil de la demeure divine, que ce soit au seuil de l'ensemble des 6 ouvertures de cette demeure ou au seuil de chaque ouverture considérée isolément, ce serpent se mord 1a queue et définit par conséquent un cercle modèle de 36 unités, subdivisible en 12 sections de 3 unités.

Une partie de la queue avalée se prolonge au-delà de la tête jusque sous la première vertèbre. Elle nous donne la mesure de l'écart entre le décompte en années lunaires (33 années lunaires) et celui en années solaires (32 années solaires) de la période luni-solaire définie. Ce qui s'en prolonge jusque dans la gorge traduit donc le déphasage de la lune par rapport au soleil.

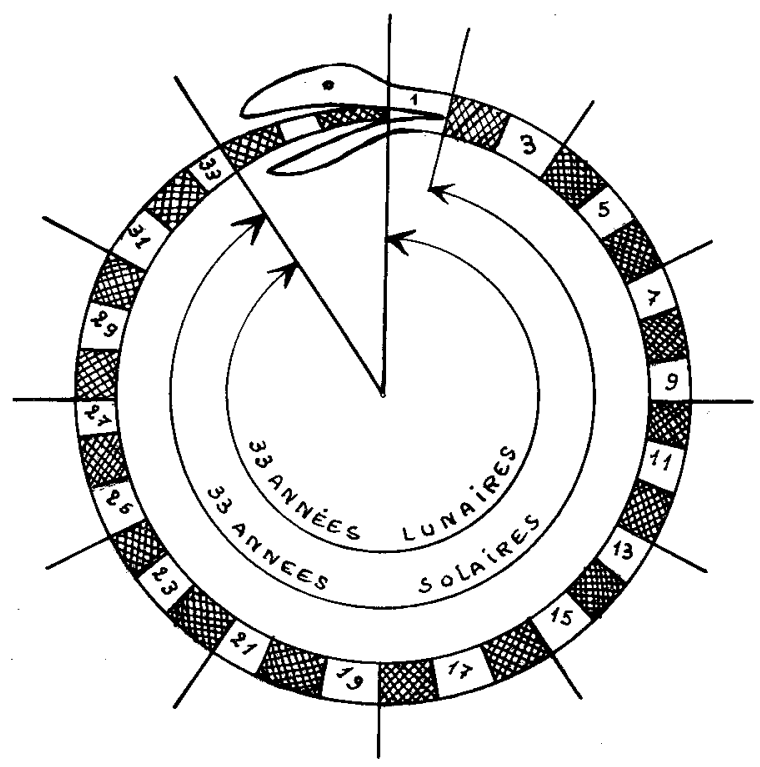

Déploiement circulaire du "serpent de la création" (tog-waog)

(14) Ce même nombre de 40 ou 41 se retrouve au premier plan en divination par afa, variété de gêomancie pratiquée au sud du Togo. 
Du fait que l'action créatrice du Soleil est qualifiée par la nature de 1'eau qu'il lui revient d'évaporer, nous sommes autorisês à considérer qu'elle émane tour à.tour d'une multitude de soleils particuliers. Et puisqu'il existe 33 catégories cycliques d'eau, il existe parallèlement 33 soleils particuliers assurant 1'approvisionnement de chacun en souffle vital. Ils sont représentés en cercle par les pierres du jizgl installé dans la cour extérieure, non loin de la sortie de I'habitation traditionnelle.

Mais ce serpent solaire essentiellement mâle, dont chaque unité de mesure vaut trois ( 3 années lunaires $=3.12=36$ lunaisons), se double secrètement d'un serpent femelle, lunaire, dont chaque même unité de mesure vaut quatre ( 4 durées de gestation de 9 lunaisons $=4.9=36$ lunaisons $)$. La colonne vertébrale de ce serpent lunaire, mesurée par le nombre de 44 (durées de gestation) et ainsi composée d'une sêrie de 44 lunes particulières, définit la période totale de fécondité théorique de la femme qui s'achève après qu'elle ait été travaillée par toutes les variêtés de lunes d'une période luni-solaire; et il dêfinit du même coup l'écart thêorique entre deux gênérations, calculé de telle sorte qu'un fils ne se marie et ne féconde sa propre femme qu'après la ménopause de sa mère (33 années lunaires après sa propre conception).

\section{La troisième immolation : celle du serpent.}

Au fur et à mesure qu'ils sont mis à contribution, les principes cycliquement ordonnés des souffles qui conduisent toute chose au monde dêploient linéairement leur activité vers le monde visible, de sorte que le serpent en activité peut être représenté linéairement sous forme de canal, couvrant toute l'êtendue du monde intermédiaire, assurant le transport jusqu'au seuil du monde visible de tout ce qui était retenu en puissance dans $1 a$ demeure divine. Demeurant fixé par la tête au lieu de l'origine, et fixé par la queue au lieu d'aboutissement, il se propose comme une échelle à 33 degrés donnant accès à toutes les richesses demeurant assoupies dans les entrailles de la terre, après que la trappe qui les cachait ait été enlevée.

Le passage de son état de repos, où $i 1$ se mord la queue en position foetale (celle du silure présenté à la vente) à son état 
d'activité où il déploie linéairement sa colonne est dû à l'action échauffante du soleil qui libère la puissance qui y était concentrée et provoque la détente spasmodique de son corps.

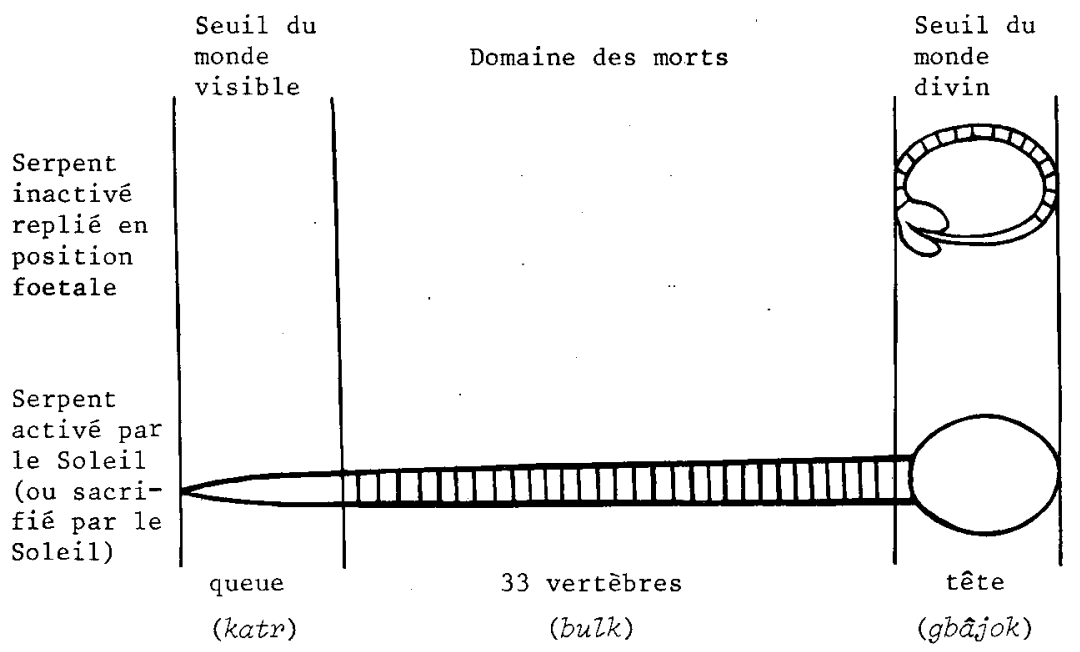

Cette action est assimilée à un sacrifice et fut même le premier de tous les sacrifices, de sorte que si la préparatrice des libations représente la Lune, l'officiant qui exécute le sacrifice visible représente d'une certaine façon le Soleil.

En chauffant la nuque du serpent replié sur lui-même, au point pris pour origine du cycle des principes qui doivent assurer l'acheminement au monde des messages, le Soleil tranche à sa manière la gorge du serpent.

Une fois que tous les messages ont été transmis, toute l'eau déversée au seuil de la demeure divine, s'étant évaporée, est allée se condenser -- elle et tous les principes auxquels elle se rattache -à la périphérie du monde visible, en sorte qu'au terme de son sacrifice, le serpent de la création se trouve reconstitué à l'autre extrémité de l'étendue spirituelle (nous avons déjà vu qu'il en était de même des "esclaves" gardiens des ouvertures, remplacés par des animaux) ; c'est pourquoi une figuration en fer de ce serpent doit faire partie des symboles attachês à la cordelette divinatoire, appelée kut, 
qui caractérise les êtres de ce niveau.

Mais s'il y a eu transfert du serpent dans un sens pour produire quelque chose au monde, il doit y avoir aussi transfert du serpent dans l'autre sens pour rapporter quelque chose à l'origine. Et la venue de 1 'eau accompagnant les bienfaits accordés grâce au sacrifice doit être compensẻe par un envoi d'eau vers l'origine.

De même que les animaux ont été sacrifiês en échange des puissances gardiennes originelles venues se reconstituer à proximité même du monde des vivants, les libations sont versées -- et le serpent de "l'eau de la création" de ce fait même sacrifié -- en échange de l'eau originelle ; puis elles représentent aussi cette eau allant communiquẹr la vie demandêe aux participants qui la consomment sous forme de bière.

Destiné à célébrer la communication de la part visible de l'être humain avec la substance invisible immuable qui assure l'identité de sa personne d'un bout à 1'autre de son existence, le sacrifice fait donc apparaître une double permutation entre des constituants du monde de 1 'origine et des constituants de 1 'environnement immédiat de 1 'habitation que s'approprient les hommes en guise de monnaie d'échange pour puiser sans risque de mort dans les trésors du monde divin et triompher de la sorte du déterminisme comme de la fatalité à laquelle reste soumise la nature ambiante.

Quelle que soit l'importance de 1'immolation des animaux, accompagnée d'un versement de sang, elle ne doit pas nous faire oublier 1 'immolation inaperçue, mais encore plus importante, du serpent, qui est le résultat du "versement d'eau".

8. Les sept "terres", ¿ l'accès contrôlé par sept serpents, devant être traversées pour venir au monde.

Nous avons pris soin de signaler que 1'équivalence de 33 années lunaires à 32 années solaires n'était valable qu'en première approximation.

Une année lunaire de plus nous aurait fourni une approximation légèrement meilleure, car 34 années lunaires équivalent à 33 années solaires avec un écart, cette fois par défaut, légèrement inférieur (valant environ 4,661 au lieu de 6,214). 
Une bien meilleure approximation nous est donc fournie par une période de $33+34=60+7=67$ années lunaires dont les écarts par défaut et par excès se compensent pour équivaloir à $32+33=65$ années solaires, avec un écart notablement réduit, mais encore insatisfaisant, d'environ 1,554 jour.

Nous n'arrivons à une approximation excellente qu'au bout d'une série de périodes alternées de $3 \dot{3}$ et 34 années lunaires, totalisant trois périodes mâles de 33 années lunaires et 4 périodes femelles de de 34 années lunaires, couvrant au total : $(34+33+34+33+34+33+34)=235$ années lunaires qui équivalent à $(4.33+3.32)=235-7=228$ années solaires, et où les écarts par excès de chaque période de 33 années lunaires sont compensés de façon très satisfaisante par les écarts par défaut de chaque période de 34 années lunaires.

Ces 228 années solaires totalisent en effet 12 cycles de 19 ans, ou "cycles de Méton" (15), valant chacun 19 années lunaires et 7 lunes, à l'issue desquels la nouvelle lune réapparaît à une date présentant un même écart par rapport à celle de l'équinoxe ou du solstice.

Le vêritable serpent est donc en fait constitué d'une succession de 235 vertèbres. Et quand il se mord la queue, les unités avalées au-delà de la tête jusque dans la gorge sont égales à 7 , mesure du déphasage entre le compte lunaire et le compte solaire.

Pour que ce grand serpent soit semblable au serpent précédent, il faudrait qu'il soit divisible en sections de valeur 7 , ayant même mesure qu'un tel déphasage et soit formé d'une succession de 11 groupes réunissant trois de ces sections. Or comme $235=11.21+7.33+4$, ce n'est possible qu'en le réduisant de 4 unités, c'est à dire en partageant la première section considérée, ou première vertèbre cervicale, en une première partie femelle, de valeur 4, et une seconde partie mâle, de valeur 3 , qui la contrôle ou la limite par en dessus, puis en admettant que la partie femelle est exclue du compte.

(15) La mesure quasi exacte en lunaisons de 19 annêes solaires (qui valent $19.12+7=235$ 1unaisons) fut attribuée à Méton et publiée en Grèce, aux Olympiades de 433 av. J.C.

235 lunaisons -19 années tropiques $=0,0864$ jour. 
Etant donné la haute valeur symbolique du nombre 33 et la signification du racourcissement ainsi opéré, une telle solution a été effectivement retenue.

Alors que le grand serpent aurait pu être décomposé en une série de 7 sections mesurant alternativement 34 et 33 unités, il a paru préférable de le décomposer en 7 sections identiques mesurant chacune 33 unités et totalisant seulement 231 unitês, quitte à faire apparaître à leur tête un segment correctif de 4 unités occupant une position singulière. Ce segment représente en effet le fondament occupé circulairement, au repos, par le serpent, c'est à dire le niveau de la substance elle-même à inonder d'eau, bref le point de départ ou point zéro, siège des principes procréateurs suprêmes, ne changeant pas lui-même de position dans la détente vertébrale provoqué par l'action sacrificielle des rayons solaires, et donc, en un certain sens, incorporable à la tête elle-même.

C'est immédiatement après lui qu'est tranchée la gorge ou la nuque du serpent pour libérer linéairement tout le reste de la colonne.

A sa suite, la seconde moitié de valeur 3 de la même vertèbre cervicale (devenant les 3 premières cervicales du premier des 7 serpents de valeur 33), prenant appui sur le fondement mesuré par 4 , nous donne la mesure du niveau initialement occupé par les puissances gardiennes mâles chargées d'empêcher la venue au monde des potentialitès inscrites dans la substance.

Pour que soit conservée au-delà des extrémités de la colonne 1 'analogie entre le grand serpent et le serpent de 33 vertèbres, 1 a mesure attribuée à sa tête doit valoir 3 fois le dêphasage entre le compte lunaire et le compte solaire, c'est à dire $3.7=21$; et $1 \mathrm{a}$ mesure attribuée à sa queue doit le valoir 4 fois, c'est à dire être égale à $4.7 .=28$. Nous le voyons alors, en position de repos, se mordant la queue, former un cercle ayant pour mesure le nombre $21+235=256=2^{8}=16^{4}$ (16).

Cependant, si nous prolongeons de part et d'autre notre progression par groupes de 33 années lunaires, nous arrivons :

(16) Nombre tout aussi éminent en divination par afa puisqu'il est celui des figures d'afa ou afadu. Toutes les figures géomantiques peuvent ainsi être inscrites circulairement le long du corps du serpent. 
-- Du côté de la tête, à compter du morceau femelle de valeur 4 laissë en dehors des 7 groupes de 33 cycles, en un point qui dépasse la tête de 8 unités.

Ces 8 unités, qui nous donnent la dimension de la langue du serpent, correspondent aux 8 cordelettes divinatoires qui servent à composer les paroles muettes fondamentales incorporées sous forme d'idées dans la tête du serpent.

-- Du côtê de la queue, en un point qui dépasse de 5 unités la dimension attribuêe à la queue, de sorte que celle-ci apparaît comme une sorte de stylet mobile inscrivant les messages qui lui sont communiqués et la mettent en branle sur la table des 5 éléments matériels.

Ce point nous mène jusqu'à l'extrême limite du vestibule du monde des vivants, au-delà de laquelle nous entrons dans le monde visible organisé.

Entre le moment de mise en activité de la série des principes conduisant toute chose au monde et le moment où s'achève l'introduction au monde, est ainsi parcourue une distance mesurée par $8.33=$ 264 unités ou, si 1 'on prêfère tenir compte du point de départ comme du point d'arrivée, par $264+2=266$ unités, nombre de jours de gestation de l'être humain depuis celui de sa conception dans le ventre de sa mère jusqu'à celui de son accouchement (17).

Cette distance est analogue à celle gravie par les défunts allant rejoindre l'origine quittée jadis par eux en prenant le chemin de la

(17) Pour le Mwaba-Gurma, est bulk (pluriel buli) tout disparu ou tout être en route vers le monde et non encore accouché. Mais dans une acception plus précise, les buli (qui en tant qu'enveloppes successives ou "peaux de la terre" sont aussi appelés tîgbana) se définissent comme les principes des diverses couches qui séparent I'être vivant du seuil du monde de 1 'origine et assurent pour $1 u i$. une médiation avec le monde de 1 'origine.

D'un point de vue approché ou limité, il en existe 33 (ou 34) puisque ce nombre est répété partout. D'un point de vue plus complet, il en existe $8.33+2=266$. Et d'un point de vue encore plus complet, tenant compte du fait que pour accéder au rang de vieux il faut avoir franchi deux générations au-delà de la naissance, ou du fait que ia semence virile transnise par les aỉeux ne parvient dans le ventre de l'épouse qu'après avoir traversé le père du mari (vieux de l'habitation) et le mari lui-même, et sachant que deux générations successives comptent en réalité pour $33+34=67$ années lunaires, il en existe $266+67=333$. 
naissance. Et puisque les dëfunts sont tous regroupés derrière leur frère aîné par générations d'une durée théorique de 33 années 1 unaire (période de fécondité d'une même femme), elle est subdivisée en couches semblables de 33 unités commandées chacune par un principe distinct qui n'est autre qu'un serpent, à vaincre ou sacrifier pour passer d'une des couches à la suivante.

Au départ de 1'origine, le sacrifice du premier serpent, qui a autorité sur la première couche, donne accès à la seconde couche ..., et le sacrifice d'un septième serpent donne accès à la huitième couche qui n'est autre que le vestibule du monde visible. Là le serpent demeure pour assurer comme il convient, en ceinturant leur habitation, la protection des êtres vivants (il n'est éventuellement sacrifié que pour amorcer cette fois le retour à l'origine).

Depuis les entrailles profondes de la terre jusqu'au monde visible, il y a donc, une fois libéré de la couche origine par le sacrifice du premier serpent, 7 autres couches à remonter pour émerger au monde visible. De plus, cette série de couches est qualifiée dans son ensemble par la portion vertébrale du serpent circulaire qui lui sert de fondement, portion qui appartient dans la plupart des cas à 1 'une ou 1 'autre des 7 sections de 33 vertèbres. Ainsi la "peau de la terre" d'oũ sort l'individu qui s'achemine vers la naissance qualifie en permanence sa dimension spirituelle mesurée par le nombre 7 .

Ce sont là les 7 "terres" de la tradition, analogues aux 7 "cieux" planétaires traversés par la Lune avant de déverser l'eau de sa calebasse, puisée au niveau de la voie lactée, sur la substance terreuse constituée au seuil de la demeure divine et analogue à la matrice de la femme.

Le principe de cette dimension spirituelle, reprêsenté par une série de vertèbres de python, n'est autre que le miq̨, ou "nez" de l'individu, encore appelé gbâjok en jargon divinatoire, par oû̀ le flux de vie lui est communiqué ou retiré. Et de même que, selon d'autres conceptions, une relation privilégiée place tout individu sous la protection de 1 'une des 7 "planètes", il est, dans la plupart des cas, en relation privilégiée avec l'une des 7 "terres" (18).

Non seulement l'ancêtre (paternel ou maternel) qui patronne la venue au monde de tout individu, et lui sert ensuite d'intermédiaire 
nécessaires pour atteindre sa substance, doit avoir même miģz que lui, mais il importe que la présentatrice d'eau enfarinée, ou sâââââ, qui doit intervenir lors d'un.sacrifice sanglant accompli en sa faveur, soit en principe aussi de même mį̧

En conclusion, il nous faut constater que les Mwaba-Gurma réexpriment périodiquement dans leurs sacrifices la conception de 1 'homme et du monde à laquelle ils sont attachẻs, et qui est $1^{\prime}$ âme même de leur société, en tant que système symbolique de référence dont s'inspirent toutes leurs institutions et leurs comportements. Par le sacrifice (dont la tête est la divination) ils recréent ou regënèrent continuellement, à partir de ses fondements, I'ensemble du tissu social. Aussi n'est-ce pas le sacrifice qui doit être expliqué par sa relation à divers autres phénomènes géographiques, économiques ou sociaux ; ce sont au contraire tous les autres phénomènes sociaux qui méxitent d'être envisagés à la lumière du sacrifice. Ceci suppose qu'il soit être étudié dans sa totalité et que soit notamment restitué à la place qui lui revient, c'est à dire à la toute première, le rôle de la présentatrice d'eau enfarinée et le symbolisme général de 1'eau.

(18) Dans la plupart des cas seulement. En effet, au dessus des 7.33 vertèbres du serpent total, il reste encore 4 vertèbres assimilées à la tête.

C'est pourquoi certains enfants doivent être demandés à Dieu à la source même et non au principe de 1 'une des 7 "terres"; on les juge envoyés au monde non par un ancêtre tutélaire de même mięz, mais par la collectivité des aỉeux éloignés rassemblés en la personne du trgban de territoire dont le rang est en correspondance avec l'audelà des 7 "terres".

I1 est d'usage de congédier les esprits psychopompes qui ont acheminé au monde 1 'un de ces enfants non pas, comme habituellement, près de son yệndu dans 1 a courette de sa mère, mais à l'extérieur de I'habitation, près du jizl. 


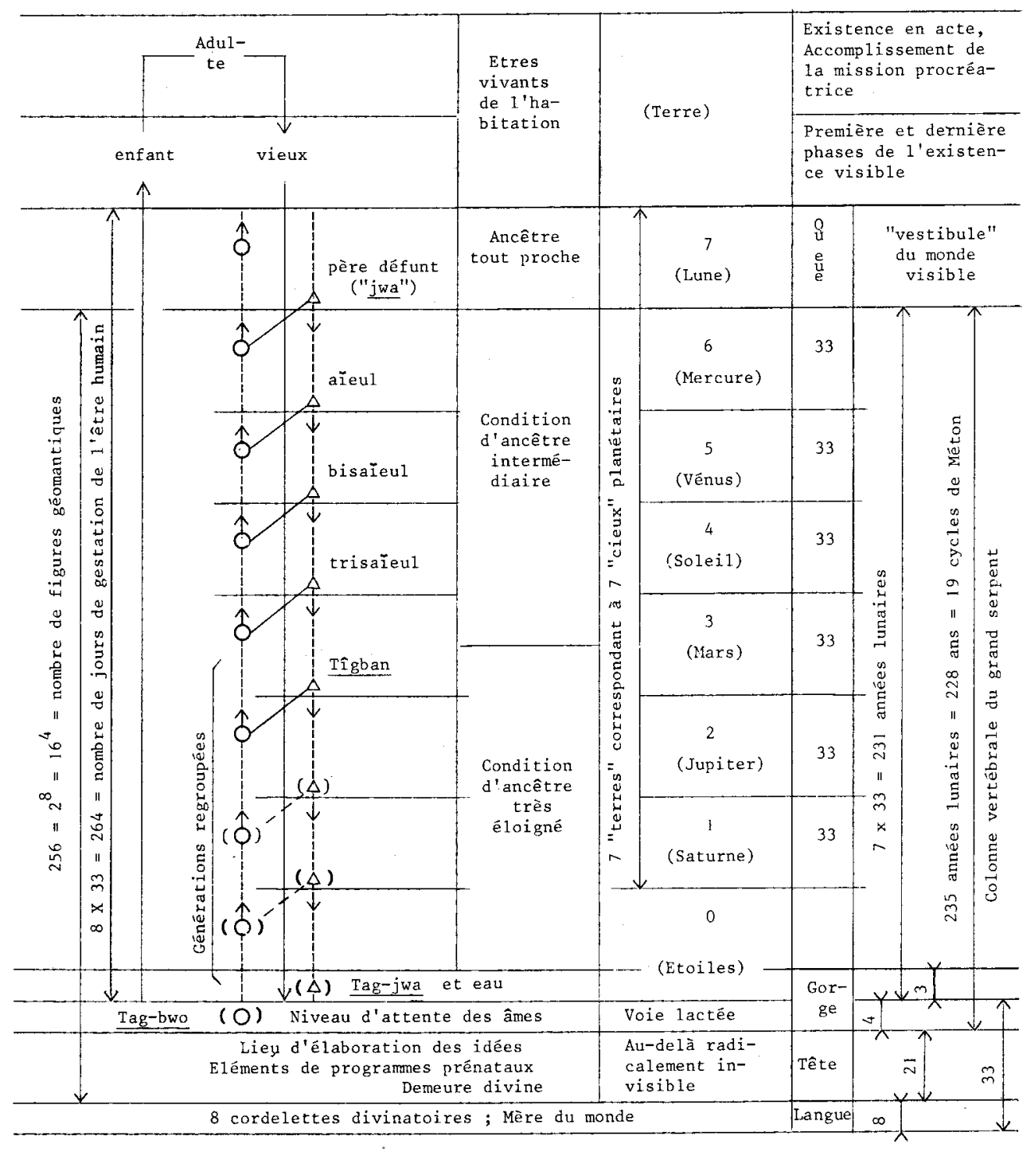

Tableau I, 15 : Déploiement linéaire du grand serpent 1uni-solaire, et sa relation aux gênérations de défunts 